\title{
乌ु \\ Multiple harmonic frequencies resonant cavity design and half-scale prototype measurements for a fast kicker
}

\author{
Yulu Huang \\ Institute of Modern Physics, Chinese Academy of Sciences, Lanzhou 730000, China; \\ Thomas Jefferson National Accelerator Facility, Newport News, Virginia 23606, USA \\ and University of Chinese Academy of Sciences, Beijing 100049, China \\ Haipeng Wang, ${ }^{*}$ Robert A. Rimmer, Shaoheng Wang, and Jiquan Guo \\ Thomas Jefferson National Accelerator Facility, Newport News, Virginia 23606, USA
}

(Received 16 September 2016; published 29 December 2016)

\begin{abstract}
Quarter wavelength resonator (QWR) based deflecting cavities with the capability of supporting multiple odd-harmonic modes have been developed for an ultrafast periodic kicker system in the proposed Jefferson Lab Electron Ion Collider (JLEIC, formerly MEIC). Previous work on the kicking pulse synthesis and the transverse beam dynamics tracking simulations show that a flat-top kicking pulse can be generated with minimal emittance growth during injection and circulation of the cooling electron bunches. This flattop kicking pulse can be obtained when a DC component and 10 harmonic modes with appropriate amplitude and phase are combined together. To support 10 such harmonic modes, four QWR cavities are used with $5,3,1$, and 1 modes, respectively. In the multiple-mode cavities, several slightly tapered segments of the inner conductor are introduced to tune the higher order deflecting modes to be harmonic, and stub tuners are used to fine tune each frequency to compensate for potential errors. In this paper, we summarize the electromagnetic design of the five-mode cavity, including the geometry optimization to get high transverse shunt impedance, the frequency tuning and sensitivity analysis, and the single loop coupler design for coupling to all of the harmonic modes. In particular we report on the design and fabrication of a half-scale copper prototype of this proof-of-principle five-odd-mode cavity, as well as the rf bench measurements. Finally, we demonstrate mode superposition in this cavity experimentally, which illustrates the kicking pulse generation concept.
\end{abstract}

DOI: 10.1103/PhysRevAccelBeams.19.122001

\section{INTRODUCTION}

To achieve high luminosity in the proposed JLEIC [1,2], an Energy Recovery Linac (ERL) based electron cooler is envisaged to cool the ion bunches (at energies of up to $100 \mathrm{GeV} / \mathrm{u}$ ) with tunable energy (up to $55 \mathrm{MeV}$ ) and magnetized electron bunches.

A relatively high electron beam current (about $1.5 \mathrm{~A}$ at 476.3 MHz repetition rate in continuous wave operation) is needed for the eventual high luminosity upgrade scheme. To reduce the technical challenges on the high current magnetized electron source, high order modes (HOM) damping in the superconducting radio frequency (SRF) ERL and high beam power at the dump, a multiturn electron circulator cooler ring (CCR) is proposed. This will recirculate and reuse the low emittance electron bunches 10-30 turns in the CCR, allowing the bunch repetition rate and beam current in the source and

\footnotetext{
*haipeng@jlab.org

Published by the American Physical Society under the terms of the Creative Commons Attribution 3.0 License. Further distribution of this work must maintain attribution to the author $(s)$ and the published article's title, journal citation, and DOI.
}

the ERL to be reduced by an equal factor (to $150 \mathrm{~mA}-50 \mathrm{~mA}$ at 47.63 MHz-15.88 MHz repetition rate). The final number of turns will be determined in practice by how long the emittance and therefore cooling rate can be maintained.

A pair of ultrafast kickers are needed for the exchange of electron bunches between the ERL and the CCR. The present JLEIC design assumes a bunch repetition frequency of $476.3 \mathrm{MHz}$. If the recirculating factor of the cooling electron bunches is 10 in the CCR, then the kicker is required to operate at a pulse repetition rate of $47.63 \mathrm{MHz}$ with a pulse width of around 2 ns. Only every 10th bunch in the CCR will experience a transverse kick at the extraction kicker and be sent back to the ERL while the rest of the bunches in the CCR must not be disturbed significantly. A new bunch in the ERL will then be kicked into this blank space by an identical injection kicker so that the cooling can continue at the same rate. Pulsed power supplies and kicker magnets with these characteristics are beyond the state of the art. An alternative method, investigated here, is to synthesize a continuous train of such short pulses by summing a sufficient number of cosine waves at harmonic frequencies of the kicking repetition frequency.

Detailed discussions on the synthesis of the kicking pulse and the comparison of different kicking schemes have 


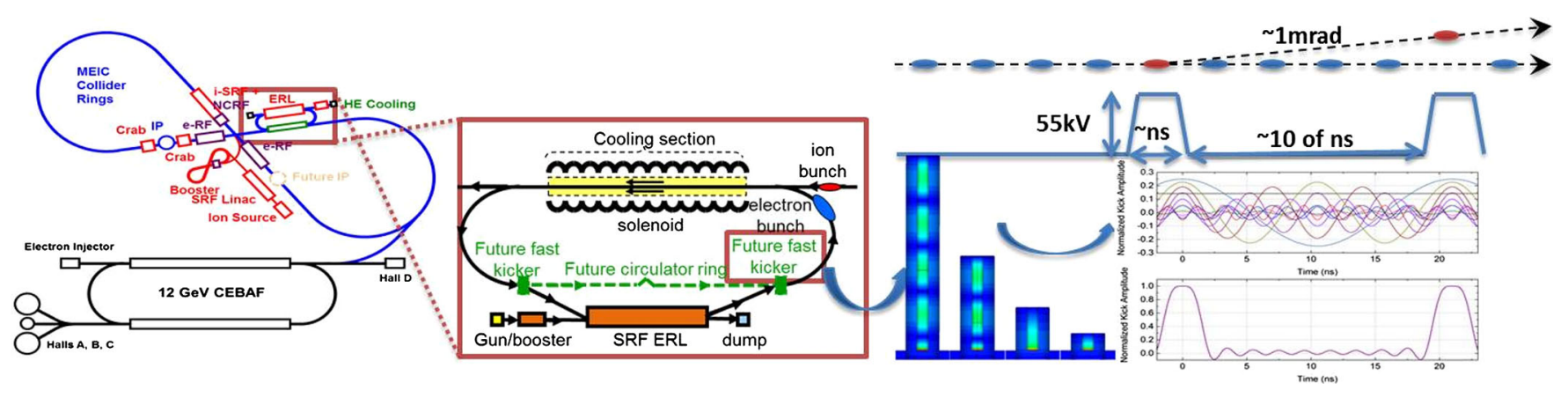

FIG. 1. The scheme of the ERL-based electron circulator cooler ring in the proposed JLEIC and the design concept of an ultrafast kicker with multiple harmonic modes generated by a group of QWRs.

been summarized in the previous published papers [3,4]. In simulations the flat-top waveform has excellent performance in conserving the transverse emittance of the cooling electron bunches during the recirculation. Such a kicking pulse can be synthesized by 10 harmonic modes of the 47.63 MHz kicker pulse repetition frequency, by using four QWR based deflecting cavities with the harmonic mode number distribution of $5: 3: 1: 1$ [5-7]. Figure 1 shows the scheme of the ERL-based electron circulator cooler ring in the proposed JLEIC and the design concept of the ultrafast kicker with multiple harmonic modes generated by a group of QWRs.

The QWR has been widely used as an accelerating cavity [8], and proposed as a deflecting and crabbing cavity [9] due to its compact structure at low frequency. In the crabbing applications, the design finally evolved into a symmetric double quarter wave structure [10] to cancel the longitudinal on-axis electric field and meet the beam dynamics requirements for multipole components. In deflecting applications, especially at very low frequency cases like this kicker cavity (47.63 MHz), an asymmetric single QWR would be more compact. However, the cavity structure near the beam pipe must be optimized to reduce the on-axis longitudinal electric field component in order to reduce beam loading in the high current application.

\section{DEFLECTING QWR DESIGN AND GEOMETRY OPTIMIZATION}

The section views of this deflecting QWR cavity model with the electromagnetic field distributions of the fundamental mode (47.63 MHz) are shown in Fig. 2. The electron bunches traveling through the cavity will be deflected by both the transverse electric and magnetic fields.

For relativistic electron bunches traveling in the $\mathrm{z}-$ direction and being deflected in the x-direction as shown in Fig. 2, the effective transverse kick voltage of the $n$th harmonic mode can be calculated from:

$V_{t n}=\int_{-\infty}^{\infty}\left[E_{x n}(z) \cos \left(\frac{2 \pi z}{\lambda_{n}}\right)+\eta_{0} H_{y n}(z) \sin \left(\frac{2 \pi z}{\lambda_{n}}\right)\right] d z$
Here $\lambda_{n}$ is the wavelength of the $n$th harmonic, and $\eta_{0}=$ $120 \pi$ is the free space impedance. The total kick voltage from all harmonic modes is

$$
V_{t}=V_{0}+\sum_{n=1}^{N} V_{t n} \cos \left(n \omega_{1} t+\varphi_{n}\right) .
$$

Here the constant term $V_{0}$ represents a DC offset which could be achieved with a small magnet in operation. $\omega_{1}$ is the bunch repetition frequency in the ERL (47.63 MHz), $N$ is the harmonic number (10 for the flat-top scheme), and $\varphi_{n}$ is the rf phase of the $n$th harmonic. $t=z / c, c$ is the speed of light.

The distributions of the field component $E_{x}, E_{z}$, and $H_{y}$ on the beam axis for the fundamental mode (47.63 MHz) and the highest mode $(428.67 \mathrm{MHz})$ in the five-odd-mode cavity are shown in Fig. 3. The magnetic field at the beam path has a reverse deflection effect to the electric field, and the fraction of magnetic field to electric field is higher for the higher harmonic modes. The integrated effect of the longitudinal electric field is zero in the deflecting phase, but in the real machine operation at high current it may lead to a significant beam induced power that must be corrected by the rf source to maintain the rf phase. It may be possible to further optimize the cavity structure near the beam pipe to reduce the longitudinal electric field.

In the following subsections, the optimization of cavity geometry to get a high transverse shunt impedance for each

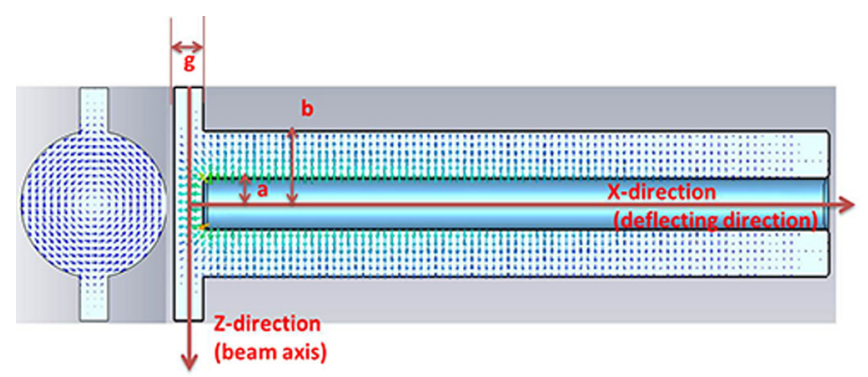

FIG. 2. Deflecting QWR cavity model of the fundamental mode $(47.63 \mathrm{MHz})$ with the left view section showing the magnetic field distribution (left) and the front view section for the electric field distribution (right). 

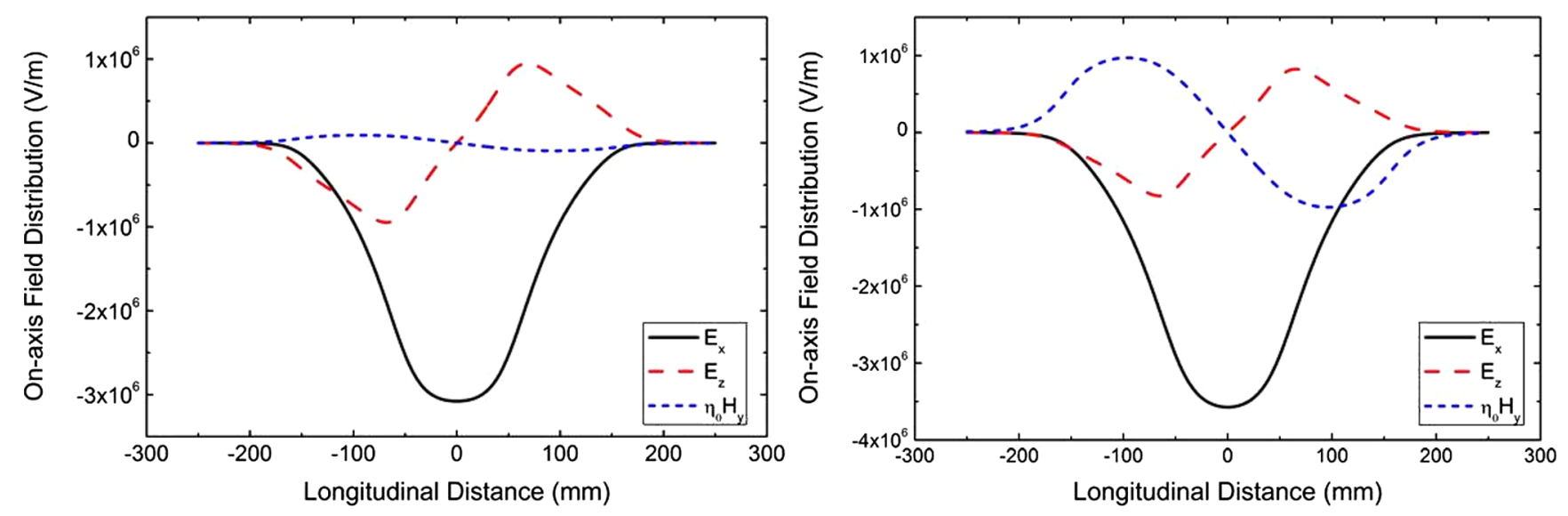

FIG. 3. Field distribution of the fundamental mode (47.63 MHz, left) and the highest mode (428.67 MHz, right) in the five-odd-mode cavity along the beam axis of the deflecting QWR. The magnetic field is multiplied by the free space impedance $\left(\eta_{0}=120 \pi\right)$ to give a fair comparison of the respective contribution of the electric and magnetic field to the transverse kick. All these electromagnetic fields are normalized to $1 \mathrm{~J}$ stored energy.

mode, using both analytical and numerical methods, the straight taper segment and the multistub tuner designs to get the cavity tuned for all resonant modes simultaneously, the loop coupler design and finally the rf power requirements will be discussed.

\section{A. Optimization of the transverse shunt impedance}

To minimize the power requirements at a given kick voltage, it is necessary to maximize the transverse shunt impedance $R_{t n}$, for each mode, which is defined as:

$$
R_{t n}=\frac{V_{t n}^{2}}{P_{c n}}
$$

Here $P_{c n}$ is the total $r f$ loss on the cavity wall for the $n$th mode.

Neglecting the effects of the beam pipe and the small reverse deflection from the magnetic field, the transverse kick voltage acquired by an on-crest particle is the integral of the time-dependent transverse electric field along the beam line [11]. For the $n$th mode, we may approximate the electric field in the end capacitor gap (width g, as shown in Fig. 2.), as a constant up to radius a, the inner conductor radius, and falling to zero at the outer conductor, radius $b$, with a fringing factor defined as:

$$
F_{n}=\frac{\int_{-b}^{b} E_{x n}(z) d z}{2 a \frac{V_{0 n}}{g}}
$$

where $V_{0 n}$ is the electric potential difference between inner and outer conductor at the end capacitor for the $n$th mode. Then the transverse kicker voltage acquired by an on-crest particle from the $n$th harmonic mode can be written as:

$$
V_{t n}=\int_{-b}^{b}\left[E_{x n}(z) \cos \left(\frac{2 \pi z}{\lambda_{n}}\right)\right] d z=\frac{2 a V_{0 n} T_{n} F_{n}}{g}
$$

where $T_{n}$ is the transit time factor for the $n$th harmonic mode:

$$
T_{n}=\frac{\int_{-b}^{b}\left[E_{x n}(z) \cos \left(\frac{2 \pi z}{\lambda_{n}}\right)\right] d z}{\int_{-b}^{b} E_{x n}(z) d z} .
$$

Without beam pipes, and neglecting the power loss in the capacitive end gap, the total power dissipation of the $n$th mode $P_{c n}$ in the cavity consists of three parts; the inner cylindrical conductor (radius $=\mathrm{a}$, length $\left.\approx \lambda_{1} / 4\right) P_{a n}$, the outer cylindrical conductor (radius $=\mathrm{b}$, length $\approx \lambda_{1} / 4$ ) $P_{b n}$, and the inductive end disk (radius from a to $\mathrm{b}$ ) between inner and outer conductors $P_{\text {endn }}$.

$$
P_{c n}=P_{a n}+P_{b n}+P_{e n d n} .
$$

The power dissipation on the inner conductor of the $n$th mode $P_{a n}$ can be calculated from:

$$
\begin{aligned}
P_{a n} & =\frac{1}{2} R_{s n} \int\left[H_{\varphi_{n}} \sin \left(\frac{2 \pi x}{\lambda_{n}}\right)\right]^{2} d S \\
& \approx \frac{1}{2} R_{s n} \int_{0}^{\frac{\lambda_{1}}{4}}\left[H_{\varphi_{n}} \sin \left(\frac{2 \pi x}{\lambda_{n}}\right)\right]^{2} 2 \pi a d x \\
& =\frac{R_{s n} \lambda_{1} I_{0 n}^{2}}{32 \pi a} .
\end{aligned}
$$

Similarly, the power dissipation on the outer conductor of the $n$th mode $P_{b n}$ can be calculated from:

$$
P_{b n}=\frac{R_{s n} \lambda_{1} I_{0 n}^{2}}{32 \pi b}
$$

and the power dissipation on the inductive end disk of the $n$th mode $P_{\text {endn }}$ can be calculated from: 


$$
\begin{aligned}
P_{\text {endn }} & =\frac{1}{2} R_{s n} \int H_{\varphi_{n}}^{2} d S \\
& =\frac{1}{2} R_{s n} \int_{a}^{b} H_{\varphi_{n}}^{2} 2 \pi r d r \\
& =\frac{R_{s n} I_{0 n}^{2} \ln (1 / \xi)}{4 \pi}
\end{aligned}
$$

where $I_{0 n}$ is the rf current of the $n$th mode on the inductive end of cavity wall, which depends on the cavity input power; $\lambda_{1}$ is the wavelength of the fundamental mode in the cavity, which also determines the cavity length; $\mathrm{x}$ is taking the inner conductor electrode center as the origin, so the integration from $x=0$ neglects the gap distance g. $\xi$ is the radius ratio between inner and outer conductors,

$$
\xi=\frac{a}{b} .
$$

$R_{s n}$ is the surface resistance of the $n$th mode,

$$
R_{s n}=\sqrt{\frac{\pi f_{n} \mu}{\sigma}}
$$

$f_{n}$ is the frequency of the $n$th mode, $\sigma$ is the conductivity of the cavity material, in this case copper, and $\mu$ is its permeability.

Then the transverse shunt impedance can be found as:

$$
R_{t n}=\frac{128 \pi(b \xi)^{2} Z_{0}(\xi)^{2} T_{n}^{2} F_{n}^{2}}{g^{2} R_{s n} \lambda_{1}\left(1 / b \xi+1 / b+8 \ln (1 / \xi) / \lambda_{1}\right)}
$$

where $Z_{0}$ is the characteristic impedance of the transmission line, which can be calculated form Eq. (14),

$$
Z_{0}(\xi)=\frac{V_{0 n}}{I_{0 n}}=\frac{\eta_{0} \ln \left(\frac{1}{\xi}\right)}{2 \pi} .
$$

From this formula it can be seen that $R_{t n}$ is larger when the gap distance $\mathrm{g}$ is small, but this gap should be big enough to stay clear of the passing beam. Higher harmonic modes will have lower shunt impedance since the surface resistance will increase with frequency and the transit time factor will decrease at the same time. The outer conductor radius $b$ is limited by the transit time factor of the highest mode (the time it takes the electron to transverse the cavity should not exceed one-half of an $\mathrm{rf}$ period so that the electron does not see a reversal of the electric field),as shown in Eq. (15).

$$
\begin{aligned}
& 2 b=\frac{\lambda_{10}}{2} \\
& \lambda_{10}=\frac{\lambda_{1}}{10} .
\end{aligned}
$$

In this kicker design the total transverse voltage is only $55 \mathrm{kV}$ so it is convenient to use the same $\mathrm{b}$ to a ratio for all four cavities, with $\mathrm{b}$ set by the highest harmonic frequency (476.3 MHz), which will simplify fabrication.

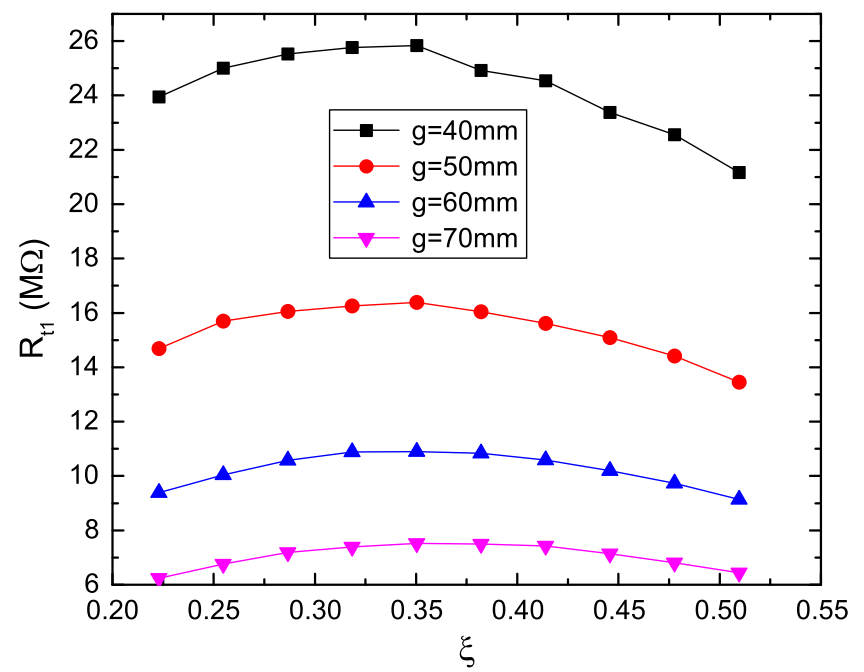

FIG. 4. Optimization of the shunt impedance of the fundamental mode for different gaps $\mathrm{g}$ when $\mathrm{b}$ is $157 \mathrm{~mm}$.

With the size of outer conductor determined, the next important thing is to optimize the ratio between inner and outer conductors. This optimization can be done numerically using CST Microwave Studio [12]. Selecting b as $157 \mathrm{~mm}$ (set by the highest mode), Rt varies at different gap value $g$ and different inner conductor ratios, as shown in Fig. 4. The optimum ratio is 0.35 , so the optimum $\mathrm{a}=55 \mathrm{~mm}$ in this situation. The shunt impedance increases rapidly with the decrease of the capacitor gap, but the optimum ratio is fairly constant over this range. In the present design the gap is fixed at $70 \mathrm{~mm}$. This optimization is based on the fundamental mode, but the trend is the same for the higher harmonic modes.

All the optimization above is based on the $\beta=1$ ultrarelativistic particles $(\mathrm{v}=\mathrm{c})$, for the $\beta<1$ situation, the cavity geometry should be adjusted to fit the velocity of the particles, Eq. (15) should be rewritten to $2 b=\beta \lambda_{10} / 2$. If the cavity geometry remains the same, the transit time factor (TTF) of each mode will be changed, the compensated power required to achieve the design kick voltage of each mode should be adjusted with the change of TTF. Figure 5 shows the TTF and the compensated power factor change with the $\beta$.

\section{B. Taper slope and tuner design}

After the cavity dimensions are determined for the lowest mode, it is crucial to make sure that every other mode is on its target frequency. Tuning of single mode QWRs is typically done by mechanically deforming a thin plate mounted at the capacitive end disk of the cavity, adjusting the distance between the bottom plate and the electrode of the inner conductor [13]. Other approaches to tune the QWR include deforming the walls of the cavity and introducing movable plungers in the magnetic field area [14]. For a multifrequency structure, the tuning is more 

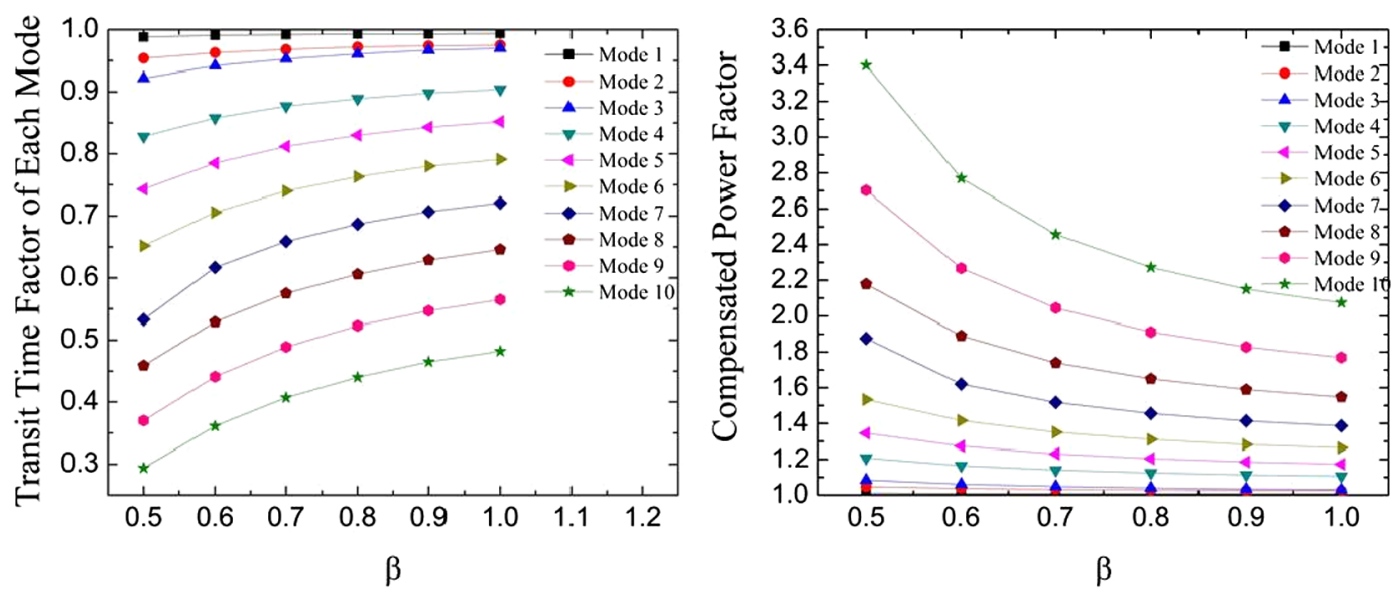

FIG. 5. Transit time factor (left) and the required compensated power (right) change with the particle velocity.

challenging since each mode cannot be tuned independently from the others. Thus a successful tuning scheme requires a number of independent tuners, equal to the number of modes, whose positions are set by a convergent iterative process.

Consider the equivalent circuit of this capacitor loaded cavity as shown in Fig. 6.

The resonant frequency can be calculated by the susceptance method. In the plane $T_{0}$, the total susceptance is zero at resonance, as shown:

$$
\sum B=\omega C_{0}-\frac{\cot \left(\frac{2 \pi l}{\lambda}\right)}{Z_{0}}=0
$$

Or:

$$
2 \pi f Z_{0} C_{0}=\cot \left(\frac{2 \pi l}{\lambda}\right) .
$$

Here $Z_{0}$ is the characteristic impedance of the transmission line in Eq. (14) and is determined by the ratio between inner and outer conductor. $C_{0}$ is the capacitor of the end gap, and $l$ is the total length of the cavity. The solutions of the equation are the resonant frequencies of the cavity which are close to odd multiple harmonics. Once the cavity

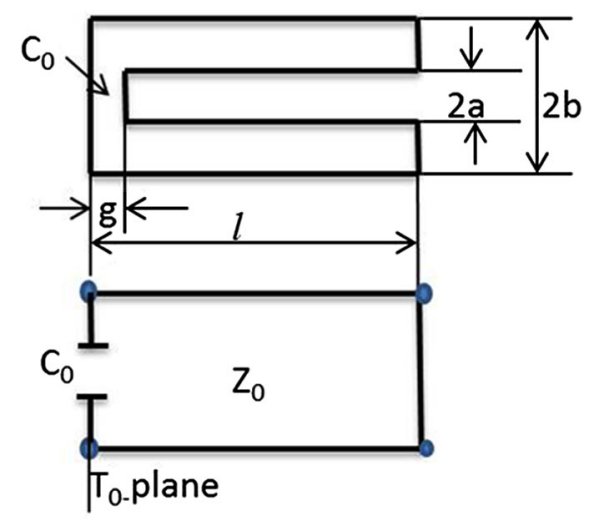

FIG. 6. The cavity schematic model (top) and the equivalent circuit of this capacitor loaded QWR cavity (bottom). length and the load capacitor are determined, the resonant frequencies are only dependent on the transmission line impedance. Thus the resonant frequencies can be tuned to harmonics by adjusting the geometry ratio $\xi$ along the cavity length, for example, tapering the cavity inner or outer wall or by inserting perturbing objects (tuners).

For this cavity, multistage tuning is adopted. Specially designed tapers on the inner conductor are used to tune the higher order modes to odd multiples of the fundamental mode. Then stub tuners on the outer conductor are used to compensate any frequency shifts due to manufacturing tolerances, temperature drifts and other perturbations during operation. The following discussions are based on the five-odd-mode cavity design and tuning as an example.

For a simple cavity model constructed as shown in Fig. 2, the cavity length can be adjusted to reduce the maximum frequency deviation of any mode from the target

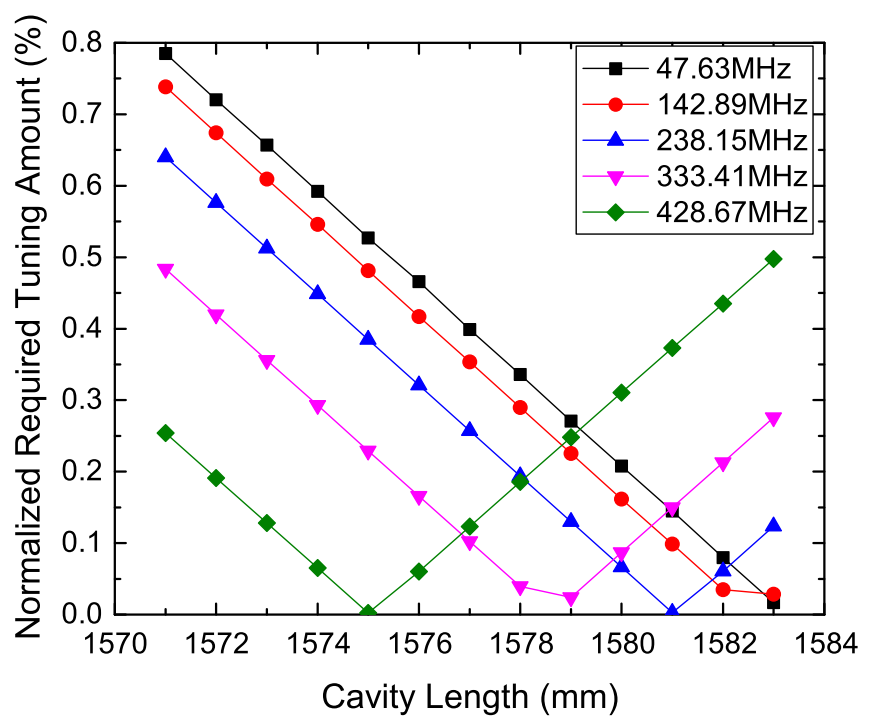

FIG. 7. Normalized required tuning amount (for tapers and stub tuners) verses cavity length, the curve inflection is due to the tuning range going to other direction from the target frequency. 
frequency. This minimizes the amount of tuning needed from the tapers and stub tuners. As shown in Fig. 7, a cavity length of $1579 \mathrm{~mm}$ requires a tuning amount from the tapers and the stubs of less than $0.3 \%$ for all modes.

In order to see the frequency response of each mode to a tuner at different locations along the cavity wall, a trial stub was inserted on the outer cylindrical wall and moved along the axial distance, as shown in Fig. 8. The frequency tuning range of the stub tuner is much less than the required tuning amount in Fig. 7, so the initial frequency offset cannot be corrected with these stubs alone. Tapers on the inner conductor are much more effective and are used in order to obtain the initial harmonic frequencies, while stub tuners are used for fine tuning.

The basic cavity structure is cylindrically symmetric along the length, and the relative frequency response of each mode to variations of taper segments on the inner conductor is similar to the tuner response on the outer conductor in Fig. 8. For the five-odd-mode cavity, five tapering points are needed. There are many possible methods to choose these taper segments and just one is presented here. As shown in Fig. 9, two end points at a1 and a5 are chosen to get the lowest tapering slopes along the cylinder while the three straight tapering transition points at a2 to a4 correspond to three nonzero frequency response points in Fig. 8 for all modes (at $530 \mathrm{~mm}, 690 \mathrm{~mm}$, $1190 \mathrm{~mm}$ ).

After the taper positions are chosen, the tapering sensitivity on each location is simulated by changing the tapering values from a1 to a5, which results in a $5 \times 5$ taper matrix $M_{\text {taper }}$, where each element $m_{i j}$ is the first order (linear) frequency response of mode $i$ to variation at the taper point $j$ in units of $\mathrm{Hz} / \mathrm{mm}$. Then the optimum taper design can be achieved by solving the taper tuning equation:

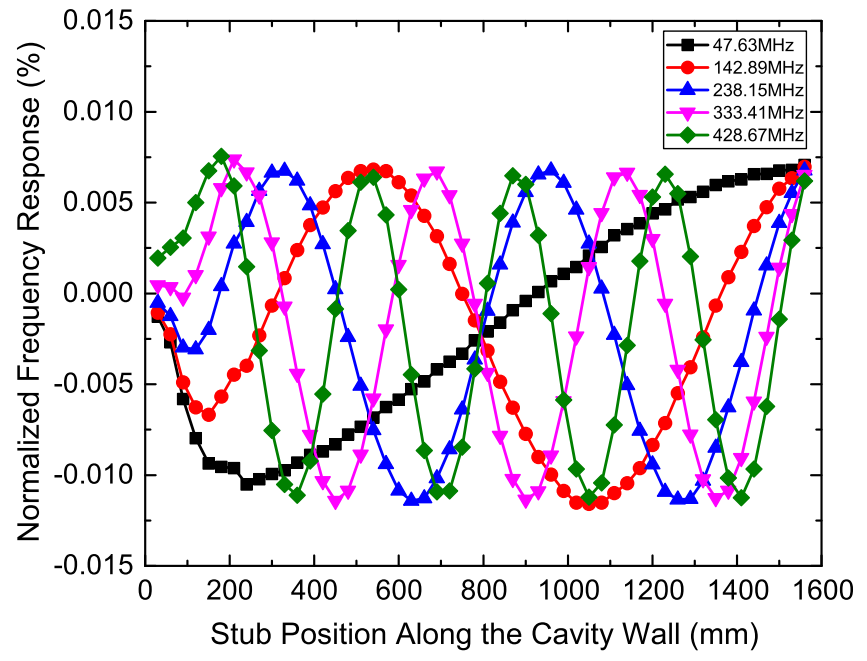

FIG. 8. Tuner position simulation for the 5 harmonics modes cavity with a cylinder trail stub radius $R=20 \mathrm{~mm}$, insertion height $H=10 \mathrm{~mm}$, perturbation moving along the outer conductor wall in the axial direction.

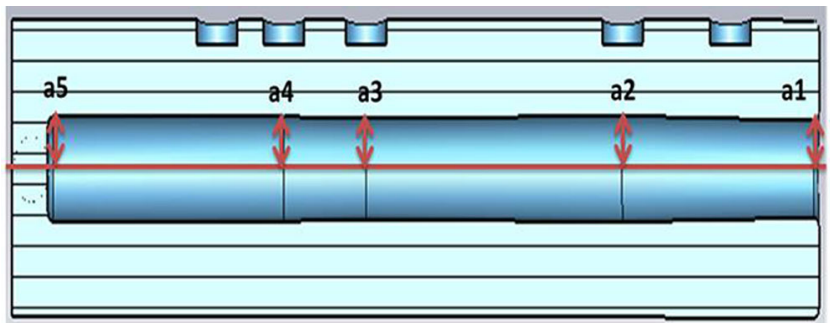

FIG. 9. Taper points chosen on the inner conductor.

$$
M_{\text {taper }} \Delta a_{n}=\Delta f_{n}
$$

where $\Delta a_{n}$ is the tapering value in $\mathrm{mm}$ at the $n$th taper point,

$$
\Delta a_{n}=\left(\Delta a_{1}, \Delta a_{2}, \Delta a_{3}, \Delta a_{4}, \Delta a_{5}\right)^{T}
$$

To avoid the unreasonable solution when solving the taper tuning equation, the taper matrix should not be ill-behaved [det(M) is not close to zero]. When choosing the taper points, one obvious criterion is to avoid the zero response points for all modes, like position $800 \mathrm{~mm}$ in Fig. 8. To provide a bi-directional tuning range by the stub tuners, the cavity tapering optimization is done with five stub tuners (80 $\mathrm{mm}$ in diameter) partially inserted (at $25 \mathrm{~mm}$ depth), as the baseline. The position of these stubs should also avoid this zero response points for all modes. Here the final designed stub diameter is double the size of the initial trail stub, thus four times of tuning range larger than the small one at a given inert length. Too much insertion for a small stub will also result in a high electric field on the stub edge. For convenience the three middle tuner positions are chosen corresponding to the middle tapering points enabling any manufacturing tolerance on the tapering point to be quickly corrected by the corresponding stub tuner. The other two are set to have good tuning sensitivity to all modes.

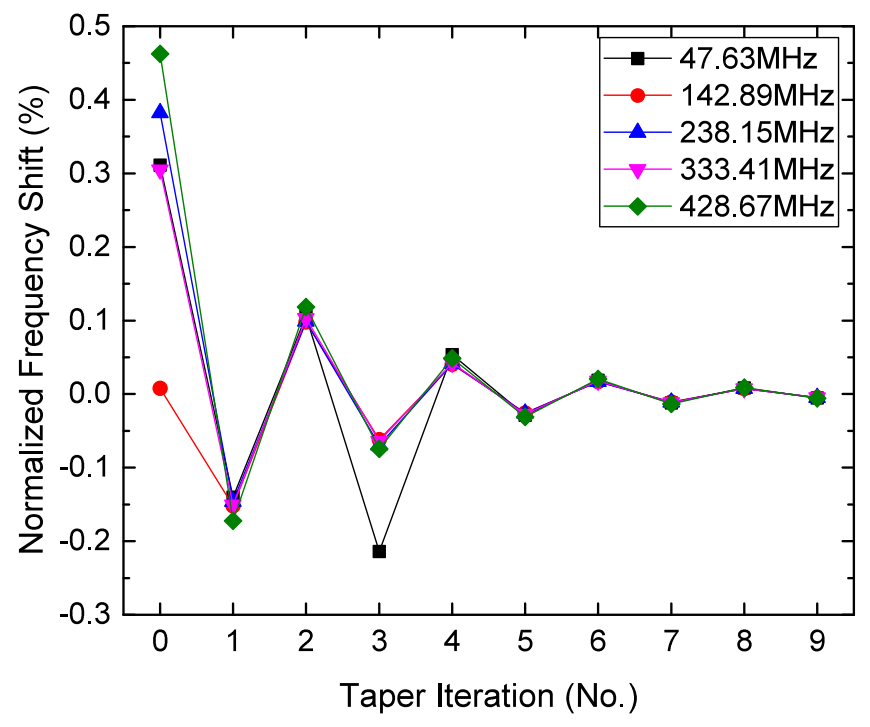

FIG. 10. Frequency convergence with taper simulation iteration number. 


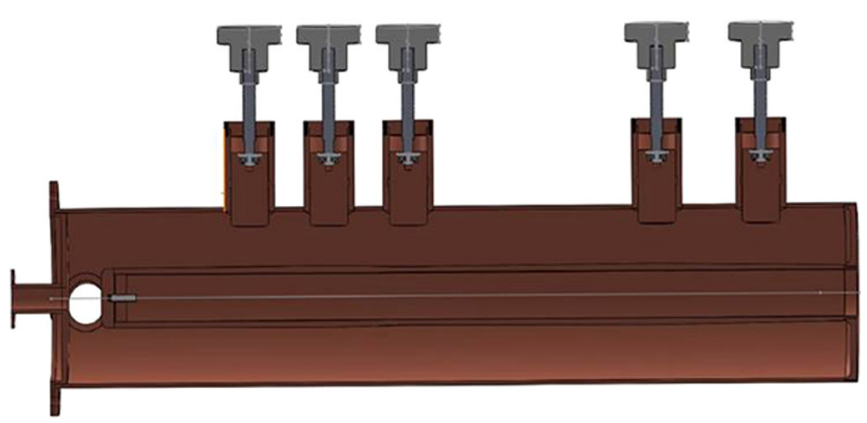

FIG. 11. A prototype cavity with five manual stub tuners for tuning five harmonic frequencies.

The frequency response to varying the tapering values is not always exactly linear, however linear approximations are used to generate the matrix and the residual error due to any nonlinearity can be eliminated by resolving the matrix equation iteratively in order to get to the target frequency within only a few cycles. The frequency convergence with taper design iterations is shown in Fig. 10.

Similarly the five stub tuners, avoiding the zero response points for all modes, at $400 \mathrm{~mm}, 530 \mathrm{~mm}, 690 \mathrm{~mm}$, $1190 \mathrm{~mm}$, and $1400 \mathrm{~mm}$ locations have been chosen $(0 \mathrm{~mm}$ is at the cavity open gap wall end), they can be used for the compensation tuning of any manufacturing errors and other perturbations during the operation such as change of the cavity temperature.

Just as for the tapering design, after the tuner positions are chosen, the tuning sensitivity of each stub is calculated by changing the insertion distance, which will result in another 5X5 tuning matrix $M_{\text {tuner }}$, the element $n_{i j}$ in this matrix means the first order frequency response of the $i$ th mode to the $j$ th tuner in units of $\mathrm{Hz} / \mathrm{mm}$. The harmonic tuning can likewise be achieved by solving the stub tuning equation:

$$
M_{\text {tuner }} \Delta h_{n}=\Delta f_{n}
$$

$\Delta h_{n}$ is the stub insertion depth value in $\mathrm{mm}$ of the $n$th tuner,

$$
\Delta h_{n}=\left(\Delta h_{1}, \Delta h_{2}, \Delta h_{3}, \Delta h_{4}, \Delta h_{5}\right)^{T} .
$$

The fields in the cavity are stronger on the inner conductor than on the outer conductor, so the frequency response to varying the tuner position is also not linear. However the error due to the nonlinearity can also be eliminated by solving the matrix equation iteratively just as was done on the taper design.

Using larger diameter tuner stubs can produce more linear matrix elements for the same tuning range, but a few iterations are still needed to get to the target frequencies. A half scale prototype cavity with 5 modes $(95.26 \mathrm{MHz}$ for the fundamental mode) has been fabricated in copper, with 5 manual stub tuners of the same material, designed for performing bench measurements, as shown in Fig. 11.

\section{Coupler design}

Two methods have been explored to drive this harmonic cavity [15]. First, the harmonic frequencies can be generated by a frequency divider after a signal source at the bunch repetition frequency, and these harmonic signals can then be filtered, amplified, and combined at low level with a precise phase delay at each harmonic channel and then fed into a wide band amplifier. Second, several narrow band amplifiers can be used at high power level, and their outputs can then be phase shift adjusted before combining together and sending into the cavity. Any combined technique has to consider building a circulator to prevent reflected power from any mismatch for any mode. Then the beam phase relative to the bunch repetition frequency can be precisely controlled and tuned. The rf power can be fed into the cavity through a single loop coupler in the high magnetic field area. For the $55 \mathrm{MeV}$ electron bunches at 1 mrad kick angle, with the flat-top kick waveform of this optimized cavity design, the dissipated power on the copper cavity wall in each mode is listed in Table I.

At such modest power any reflections due to small mismatches can be absorbed in the circulator matched load.

TABLE I. Calculated electromagnetic parameters of each mode for room temperature copper.

\begin{tabular}{lccccr}
\hline \hline Cavity & $\begin{array}{c}\text { Operation } \\
\text { Frequency }(\mathrm{MHz})\end{array}$ & $Q_{0}$ & $\begin{array}{c}\text { Flat-Top Kick } \\
\text { Voltage }(\mathrm{kV})\end{array}$ & $\begin{array}{c}\text { Transverse Shunt } \\
\text { Impedance }(\Omega)\end{array}$ & $\begin{array}{r}\text { Dissipated } \\
\text { Power (W) }\end{array}$ \\
\hline \multirow{3}{*}{ Five-Mode Cavity } & 47.63 & 8586 & 13.711 & $7.527 \mathrm{E} 6$ & 24.98 \\
& 142.89 & 14689 & 10.532 & $3.954 \mathrm{E} 6$ & 28.05 \\
& 238.15 & 18973 & 5.503 & $2.916 \mathrm{E} 6$ & 10.39 \\
& 333.41 & 22472 & 0.630 & $1.859 \mathrm{E} 6$ & 0.21 \\
Three-Mode Cavity & 428.67 & 25536 & -2.432 & $1.131 \mathrm{E} 6$ & 5.23 \\
& 28.26 & 12002 & 12.462 & $1.080 \mathrm{E} 7$ & 14.38 \\
One-Mode Cavity & 476.78 & 20784 & 2.917 & $4.082 \mathrm{E} 6$ & 2.08 \\
One-Mode Cavity & 190.52 & 27056 & -3.011 & $1.560 \mathrm{E} 6$ & 5.81 \\
DC Offset & 381.04 & 15298 & 8.129 & $1.153 \mathrm{E} 7$ & 5.73 \\
Total & & 19435 & -1.209 & $8.861 \mathrm{E} 6$ & 0.17 \\
\hline \hline
\end{tabular}




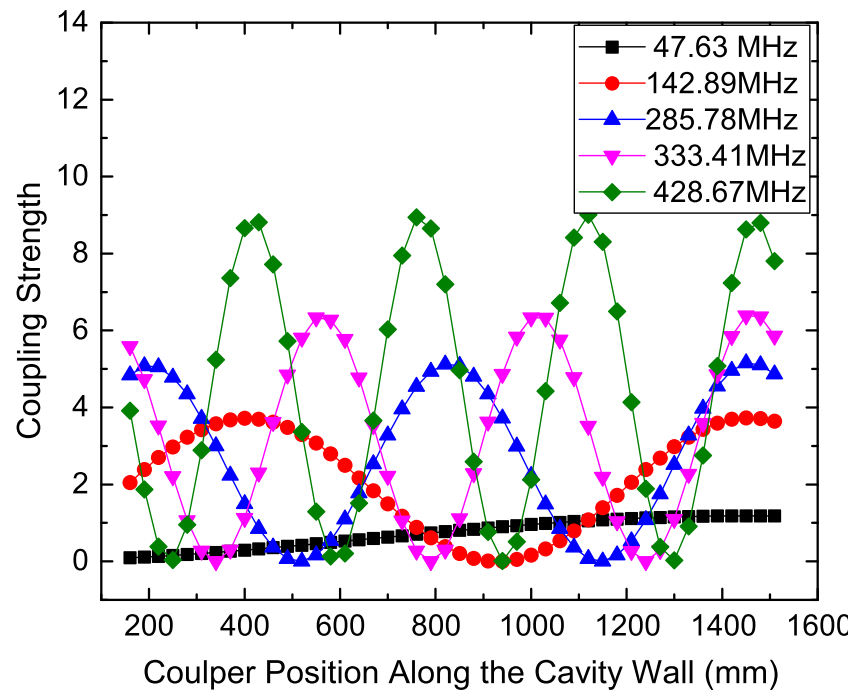

FIG. 12. Coupling strength for each mode at different locations along the cavity wall. Coupler loop size and orientation are fixed in this simulation.

One common power coupler can then be used for all harmonic modes in each cavity. The fundamental mode always has the lowest coupling strength so the loop size, rotation angle and the position (at about $1360 \mathrm{~mm}$ ) can be designed to adjust the fundamental mode to be critically coupled. The higher harmonic modes are then slightly overcoupled, as shown in Fig. 12.

\section{Multipacting}

Basically, the initial multipacting analysis in this deflecting QWR cavity can be approximated to straight coaxial lines in standing wave operation and done by the scaling laws which were described in [16]. Two different types of multipacting can be recognized, the one-point multipacting of different order on the outer conductor, and the two-point multipacting from the outer conductor to the inner conductor.

For the one point multipacting scaling calculations, the peak surface electric fields for all these 10 deflecting modes are extracted from the CST simulation results, and then be normalized and scaled to the field levels at their design kick voltages described in Table 1. With these scaled peak surface electric fields and the scaling laws, the one point multipacting orders for all modes can then be predicted. From the calculation results, only the first two modes have the risk of one point multipacting. For the first mode $(47.63 \mathrm{MHz})$, first order multipacting could occur during the field level rise (at about the $49 \%$ of the operation field level). For the second mode $(95.26 \mathrm{MHz})$, only third order multipacting could occur at the operation electric field level. Above the third mode (142.89 MHz), the possibility of multipacting is very low due to the multipacting order is higher than six.
For the two point multipacting scaling calculations, with the scaled peak surface electric fields and the scaling laws, only the first mode has the risk of multipacting during the power rise (at about $26 \%$ of the operation power level).

Further multipacting simulations on the open gap and power coupler area with 2D and 3D codes are still under the simulation study.

\section{E. High order modes}

Beam passing through the cavity, high order modes are exited. These beam-induced modes will cause significant rf power loss, and affect the following bunches' beam quality in the high current situation. These beam-induced modes are determined by the time-structure of the passing bunches. In some intense pulsed machines, such as Spallation Neutron Source (SNS) and European Spallation Source (ESS) that have multiple beam time-structures, each time structure will generate resonance excitations [17]. In our case, the electron beam has a single time-structure (continues electron bunches at $476.3 \mathrm{MHz}$ repetition rate), then only integer multiples of the bunch repetition frequency will be exited, such as 476.3 MHz, 952.6 MHz, 1428.9 MHz and so on. In our case, the electron beam has a single time-structure (continues electron bunches at $476.3 \mathrm{MHz}$ repetition rate), then only integer multiples of the bunch repetition frequency will be exited, such as $476.3 \mathrm{MHz}, 952.6 \mathrm{MHz}, 1428.9 \mathrm{MHz}$ and so on.

The beam power loss for these trapped lower order modes (LOMs), deflecting working modes (DWMs) and higher order modes (HOMs) can be calculated from the longitudinal shunt impedance and loaded $\mathrm{Q}$ of each mode. In this QWR cavity structure, there is no LOM. First considering the DWMs, without damping, the total beam power loss for these $10 \mathrm{DWMs}$ is $190 \mathrm{~kW}$ for the beam current of $1.5 \mathrm{~A}$ due to the nonzero longitudinal shunt impedance, and the major loss is on the beam repetition rate (476.3 MHz). If the cavity geometry can be optimized to get zero longitudinal shunt impedance on the highest working mode, the total beam loading power then can be only $0.027 \mathrm{~W}$ from the other nine working modes. Two methods were under investigation to do such optimization. One is to adjust the geometry of the electrode near the beam pipe to get symmetric electric field distribution for the 476.3 $\mathrm{MHz}$ mode, the other one is to change the second cavity that housing the highest mode to a double QWR structure which has nearly perfect zero longitudinal shunt impedances for all modes.

Considering the HOMs, we choose the 5-odd-mode cavity as an example. Without damping, the total beam power loss for the first 100 modes is only $8.21 \mathrm{~W}$ for the beam current of $1.5 \mathrm{~A}$. All HOMs are safely designed to fall between the beam harmonic excitation frequencies even with some tolerable cavity fabrication error. The power loss will increase dramatically if intentionally to push some modes to the resonances. It seems to us that no additional 
HOM coupler is needed for the damping but only additional cooling for the copper cavities. Further HOM analysis using the wakefield method is under the way.

\section{HALF SCALE PROTOTYPE AND RF MEASUREMENT RESULTS}

To validate the electromagnetic performance of this design, a half scale prototype with 5 odd harmonics of 95.26 MHz has been constructed. The full cavity model and associated components are shown in Fig. 13.

The outer conductor is $809 \mathrm{~mm}$ (31.85 inch) long, $155.6 \mathrm{~mm}$ (6.125 inch) OD and $148.5 \mathrm{~mm}$ (5.845 inch) ID, and was fabricated from a commercial copper water pipe stock. The nominal composition of this pipe is $99.9 \%$ copper and $0.02 \%$ phosphorus and the electrical properties are more than adequate for this purpose. Tests showed that it could also be electron beam welded without any problems.

Five tuner ports, two beam pipes, and one coupler pipe are all made from $50.8 \mathrm{~mm}$ (2 inch OD) and $38.1 \mathrm{~mm}$ (1.5 inch) ID C101 (oxygen free) copper tube. A T-Slot aluminum fixture was fabricated for assembly and alignment during the electron beam welding of the five tuner ports to the cavity outer conductor.

The inner conductor is made from a $54.0 \mathrm{~mm}(2.125$ inch) OD and $41.3 \mathrm{~mm}$ (1.625 inch) ID C101 copper tube. The four straight taper slopes on the inner conductor surface were NC machined to get the harmonic frequencies. A copper plate of $41.3 \mathrm{~mm}$ (1.625 inch) diameter with a $6.35 \mathrm{~mm}(1 / 4 \mathrm{inch})$ diameter threaded hole in the center was welded to the electric-field end of the inner conductor so a demountable electric-end cap can be connected to the inner conductor. This welded plate can also be used to align the inner conductor during the electron beam welding of the magnetic end flange.

The capacitive-end flange with the pickup port is bolted to the cavity end flange, which also allows the possibility of optimizing of the end gap structure near the beam pipe. The inductive end joints were electron beam welded for good electrical conductivity.

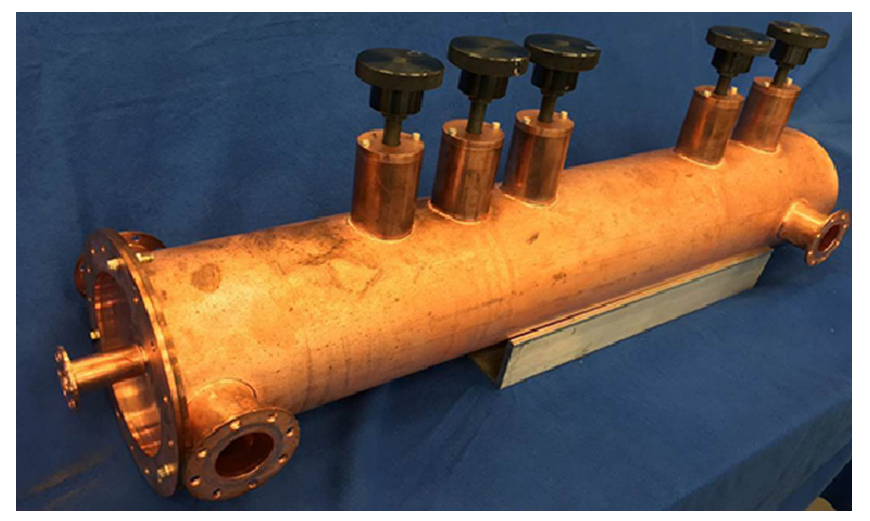

FIG. 13. Prototype cavity made of copper.
TABLE II. Resonant frequency measurements.

\begin{tabular}{lcc}
\hline \hline $\begin{array}{l}\text { Target Frequency } \\
(\mathrm{MHz})\end{array}$ & $\begin{array}{c}\text { Measured } \\
\text { Frequency }(\mathrm{MHz})\end{array}$ & $\begin{array}{c}\text { Frequency } \\
\text { Shift }(\%)\end{array}$ \\
\hline 95.26 & 95.420 & +0.17 \\
285.78 & 285.950 & +0.06 \\
476.3 & 476.785 & +0.10 \\
666.82 & 666.906 & +0.01 \\
857.34 & 857.827 & +0.06 \\
\hline \hline
\end{tabular}

The manual stub tuner design is similar to a CEBAF waveguide stub tuner. The tuning sensitivity is $1.27 \mathrm{~mm}$ (0.05 inch)/turn, set by the chosen threaded rod. Bearings are used to minimize friction between the stubs and tuner ports. Rf fingers are fitted into grooves in the tuner port to get good rf contact.

With this prototype cavity, the resonant frequency, tuning sensitivity, and unloaded quality factor have been measured for all modes. Bead-pull measurements with dielectric and metallic perturbing objects have been made and all these results compared with the numerical simulations. A demonstration of mode superposition in the cavity has also been performed.

\section{A. Resonant frequency and tuning sensitivity measurements}

The resonant frequencies of the first five harmonic modes were measured and summarized in Table II. All mode frequencies are slightly higher than the design values but the manufacturing errors are less than $0.2 \%$ for all modes.

The tuning sensitivity of each mode to each tuner was measured and summarized in the following matrix Eq. (23), the element $m_{i j}$ in the matrix means the tuning sensitivity of

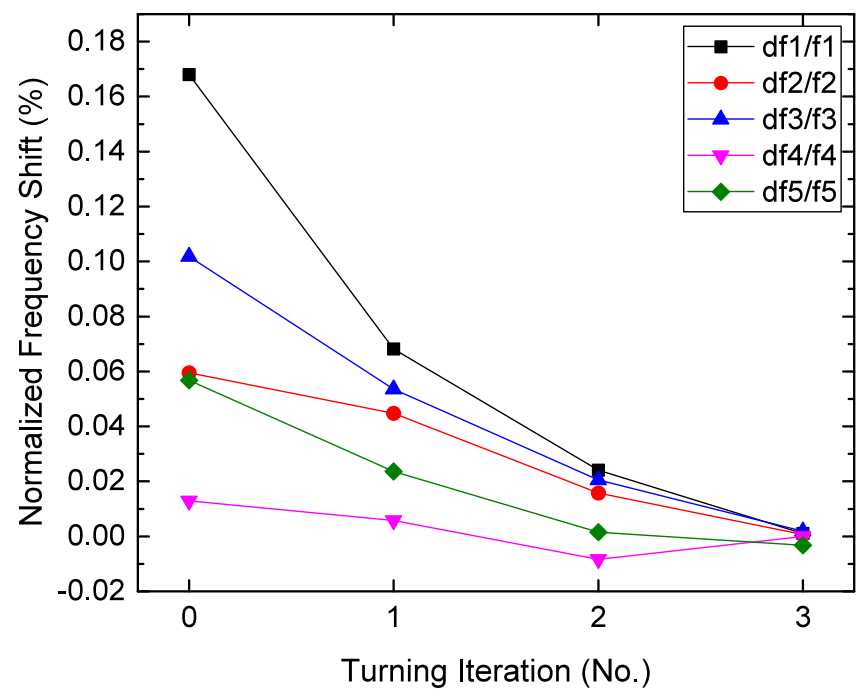

FIG. 14. Tuning process to achieve the target frequencies by solving the tuning matrix equation and adjusting the stub tuners three times in the measurement. 

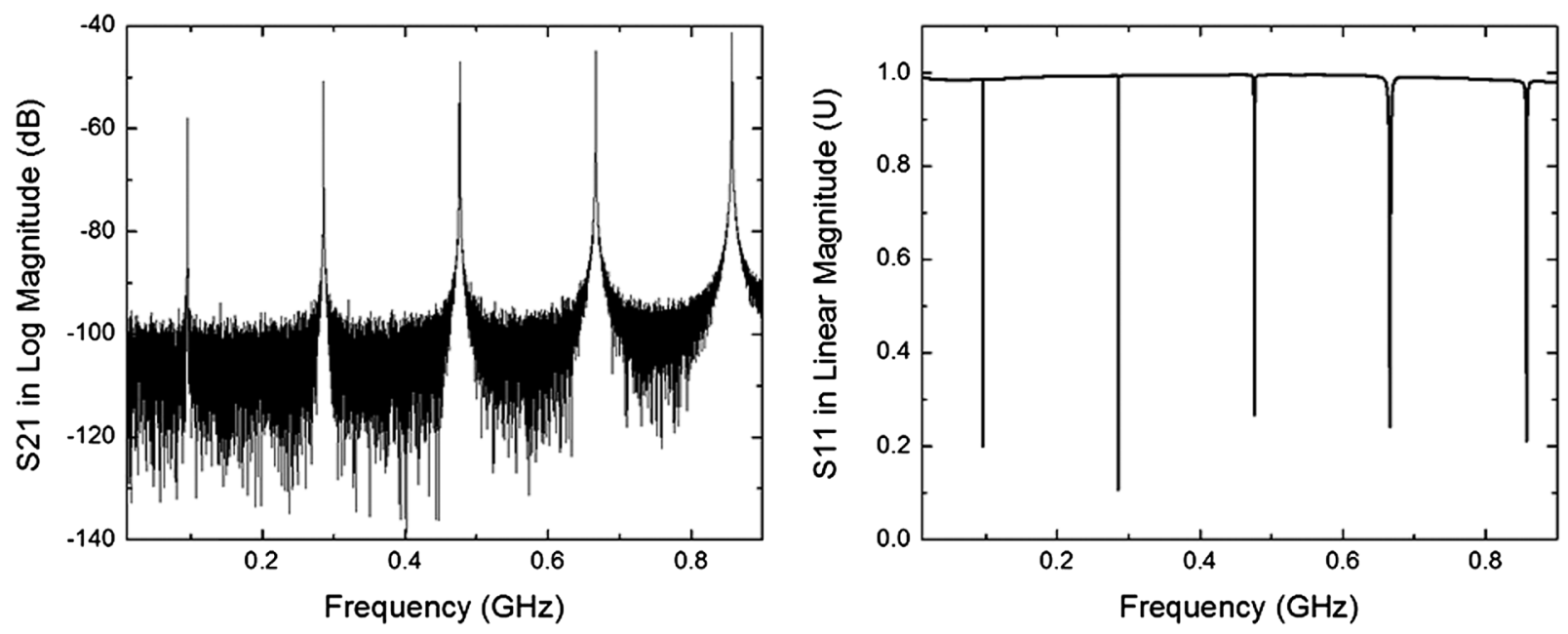

FIG. 15. The transmission coefficient between the input coupler port and the pickup port (left), and the reflection coefficient at the input coupler port (right) as a function of frequency.

the $i$ th mode to the $j$ th tuner in units of $\mathrm{kHz} / \mathrm{mm}$. Comparing to the simulation results, the maximum relative error is $0.129 \%$.

$$
M_{\text {tuner }}=\left(\begin{array}{rrrrr}
-31.26 & -26.93 & -17.80 & +3.465 & +9.370 \\
-4.803 & +33.07 & +8.583 & -100.3 & -9.134 \\
+47.09 & -126.9 & -180.2 & -150.2 & -104.3 \\
-153.5 & -216.8 & +74.25 & +20.08 & -265.5 \\
-404.6 & +97.17 & -401.0 & +14.33 & -422.1
\end{array}\right)
$$

With this tuning matrix, the frequencies were tuned to the targets with only three iterations, as shown in Fig. 14.

\section{B. Transmission and reflection coefficients, unloaded quality factor $Q$ measurement}

After the tuning process, the transmission coefficient between the input coupler port and the pickup port was measured and plotted in Fig. 15 (left), and the reflection coefficient at the input coupler port is shown in Fig. 15 (right).

The unloaded quality factors of the five harmonic modes were measured and compared with the CST simulation in Table III. The unloaded $Q$ factors are lower than the simulation values for all modes, and this difference may be caused by imperfection of the copper material, joint losses and rf contact losses at the stub tuners, but the values are quite acceptable for this experiment.

\section{Bead-pull measurement}

From the CST numerical simulations, the distribution of different field components along the beam axis can be plotted, as shown in Fig. 16. All field components are normalized to the stored energy and frequency. The dominant field component is the deflecting electric field Ex (top left in Fig. 16) the longitudinal electric field Ez (top right in Fig. 16) is much smaller than the deflecting field (maximum 10\% in magnitude squared). The transverse magnetic field Hy (bottom in Fig. 16) is much weaker, and much smaller for the lowest harmonic mode $(0.1 \%$ in magnitude squared) and relatively larger for the highest harmonic mode (6\% in magnitude squared).

The electromagnetic field distribution and mode orientation in the cavity can be obtained by a bead-pull technique, which is based on the Slater theorem [18]. In the measurement, a small metallic or dielectric object attached to a string is pulled through the rf structure, while a small amount of rf power is fed into the structure at the frequency of the resonant mode [19]. The frequency shift due to the insertion of the perturbing object is measured during the passage of the bead. The relative change in resonant frequency is proportional to the relative change in stored energy and can be expressed as Eq. (24) as a function of the local electric and magnetic field strength [20]:

$$
\frac{\Delta \omega}{\omega}=-F_{E} \frac{\varepsilon_{0} E^{2}}{U}+F_{H} \frac{\mu_{0} H^{2}}{U}
$$

TABLE III. Unloaded quality factors from the simulation and measurement.

\begin{tabular}{lccc}
\hline \hline Mode $(\mathrm{MHz})$ & CST & Measurement & Error \\
\hline 95.26 & 5665 & 5301 & $-6.43 \%$ \\
285.78 & 9770 & 9277 & $-5.04 \%$ \\
476.3 & 12531 & 11282 & $-9.96 \%$ \\
666.82 & 14834 & 13152 & $-11.34 \%$ \\
857.34 & 17109 & 14095 & $-17.62 \%$ \\
\hline \hline
\end{tabular}



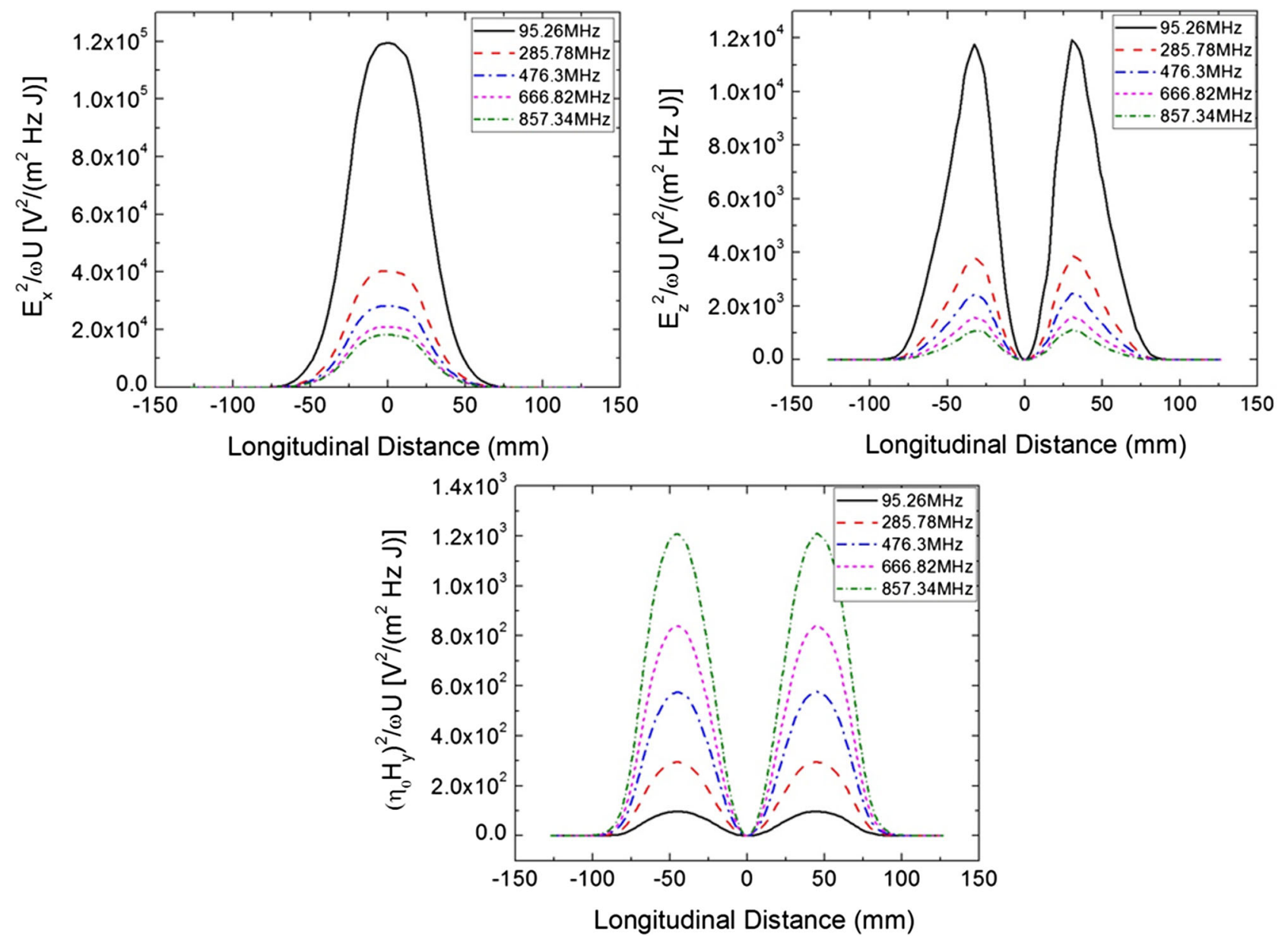

FIG. 16. On-axis field magnitude square of the different components of the electric field $E_{x}$ (top left), $E_{z}$ (top right) and the magnetic field $H_{y}$ (bottom). The magnetic field is multiplied by the free space impedance $\left(\eta_{0}=120 \pi\right)$ to give a fair comparison of the respective contribution of the electric and magnetic field to the transverse kick. All these electromagnetic fields are normalized to $1 \mathrm{~J}$ stored energy.

where $\Delta \omega=\omega-\omega_{0}$, and $\varepsilon_{0}$ and $\mu_{0}$ are the dielectric and magnetic constants of the free space, $\mathrm{U}$ is the stored energy in the cavity, and $F_{E}, F_{H}$ are the geometry factors associated with the bead shape, dimension and couplings to the $\mathrm{E}$ field and $\mathrm{H}$ field. The geometry factors for the sphere are listed in Table IV, $\varepsilon_{r}$ is the relative dielectric constant of the bead. For a perfect sphere, the form factors are the same in the longitudinal and transverse directions.

The frequency shift in the cavity resonance can be obtained with a network analyzer from the peak of the absolute S21 measurement. But for small perturbations it is

TABLE IV. Perturbation factors for sphere (radius $=a$ ).

\begin{tabular}{lcc}
\hline \hline Bead & $F_{E}$ & $F_{H}$ \\
\hline Dielectric Sphere & $\pi a^{3}\left(\frac{\varepsilon_{r}-1}{\varepsilon_{r}+2}\right)$ & \\
Metallic Sphere & $\pi a^{3}$ & $\frac{1}{2} \pi a^{3}$ \\
\hline \hline
\end{tabular}

more convenient to measure the phase shift instead of the frequency shift [21,22] when the network analyser is locked to the drive frequency, as shown in Eq. (25).

$$
\frac{\Delta \omega}{\omega}=\frac{1}{2 Q_{L}} \tan (\varphi)
$$

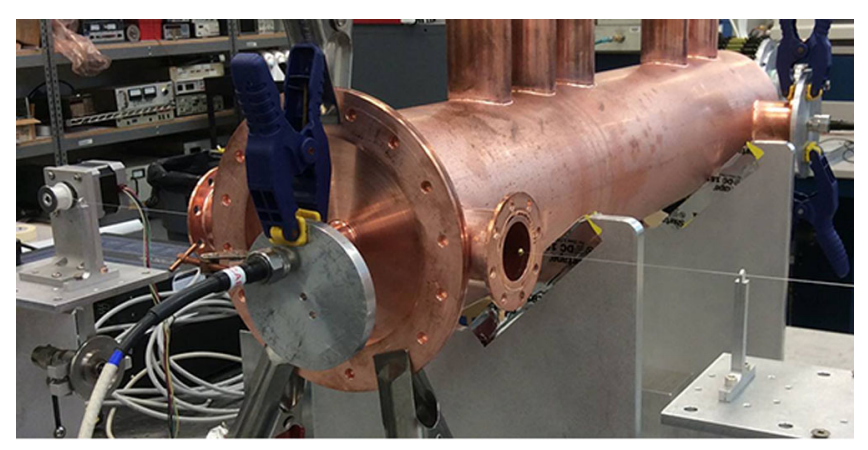

FIG. 17. Complete bead pull measurement setup. 


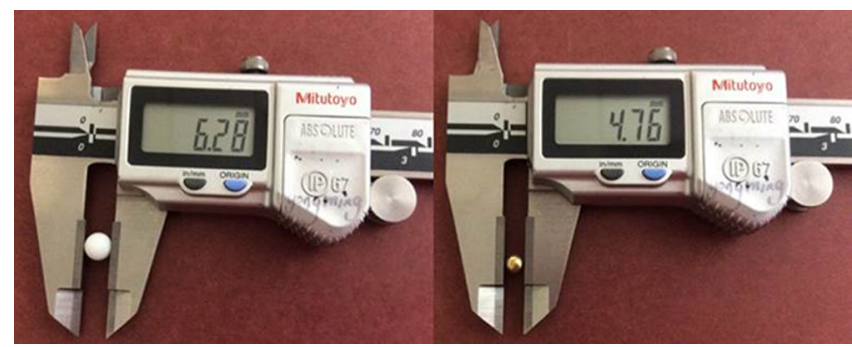

FIG. 18. Perturbing objects employed in the measurement with their dimensions measured by caliper. (Left: Teflon. Right: Brass).

where $Q_{L}$ is the loaded quality factor of resonance and $\varphi$ is the phase of the transmission coefficient between two ports coupled to the field in the cavity. When $\varphi$ is much smaller than 45 degrees, $\tan (\varphi)$ can be approximated to $\varphi$.

The complete measurement setup is shown in Fig. 17. Stepping motors controlled by a LabVIEW [23] program on a PC are used to position the bead. A transverse alignment system consisting of $\mathrm{X}-\mathrm{Y}$ stage stepping motors is installed which allows the bead position be controlled separately in the $\mathrm{X}$ and $\mathrm{Y}$ directions at each end of the cavity beam pipe. With this system, it is relatively easy to do the measurement accurately on the axis and at various offsets. An Agilent E5071C network analyzer that is interfaced by a GPIB device to the PC is used to measure the frequency shift or phase shift. A broadband amplifier is employed to increase the rf signal to noise ratio.

Two types of perturbing objects were used in the measurement, a small dielectric (Teflon) sphere and a small metallic (brass) sphere, as shown in Fig. 18. With the dielectric bead, the electric field in all directions can be picked up, and with the metallic bead, both the electric and magnetic fields will be measured.

The beads used are not perfectly spherical, so an aluminum pillbox cavity is employed to calibrate the form factor, as shown in Fig. 19. The resonant mode used here is TM010. By pulling the bead in the longitudinal direction

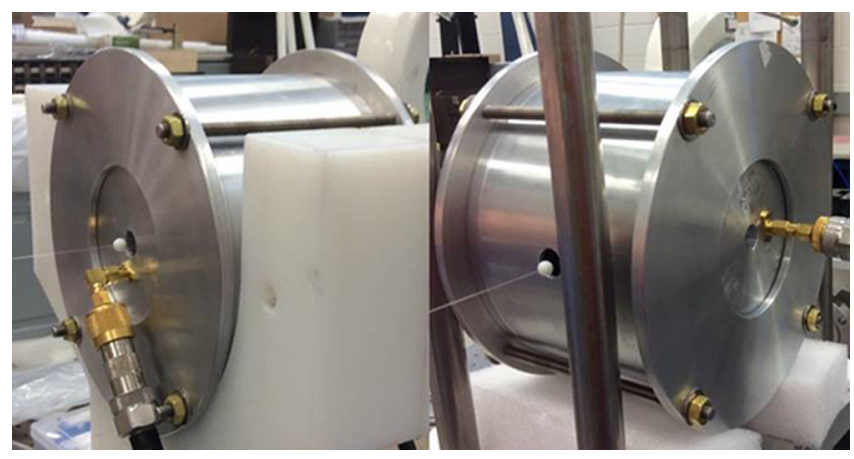

FIG. 19. Pillbox cavity used for calibration of the perturbing beads.
TABLE V. Form factors obtained by calibration.

\begin{tabular}{lccc}
\hline \hline Bead & $\begin{array}{c}\text { Transverse } \\
F_{E x}\end{array}$ & $\begin{array}{c}\text { Longitudinal } \\
F_{E z}\end{array}$ & $\begin{array}{c}\text { Transverse } \\
F_{H y}\end{array}$ \\
\hline $\begin{array}{l}\text { Dielectric } \\
\text { Sphere }\end{array}$ & $2.21 \mathrm{E}-8$ & $2.23 \mathrm{E}-8$ & \\
Metallic Sphere & $3.60 \mathrm{E}-8$ & $3.18 \mathrm{E}-8$ & $1.91 \mathrm{E}-8$ \\
\hline \hline
\end{tabular}

(left), through the known field the longitudinal form factor can be calibrated, and by pulling the bead in the radial direction (right), the transverse form factor can then be calibrated. The form factors obtained by calibration are listed in Table V. Since the beads are not perfect spheres and there is a finite through hole at the bead center, the form factors are different in the two directions.

With the calibrated beads, the final measurement results are summarized in Fig. 20. The longitudinal electric field and the magnetic field are too weak to be separated out.

\section{Mode combination experiment}

The half scale multiharmonic cavity was also used to demonstrate a simple multiharmonic frequency

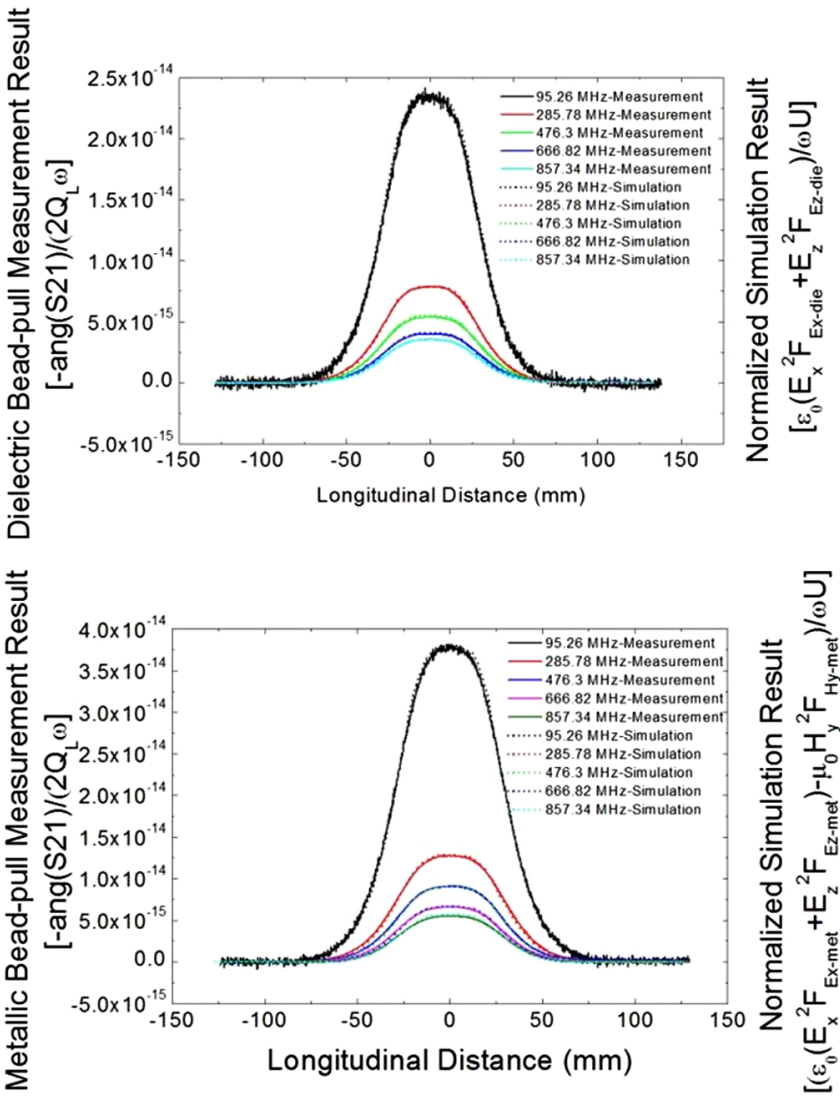

FIG. 20. Result of the measurement with the dielectric (top) and the metallic (bottom) bead, and the comparison with simulation result. 

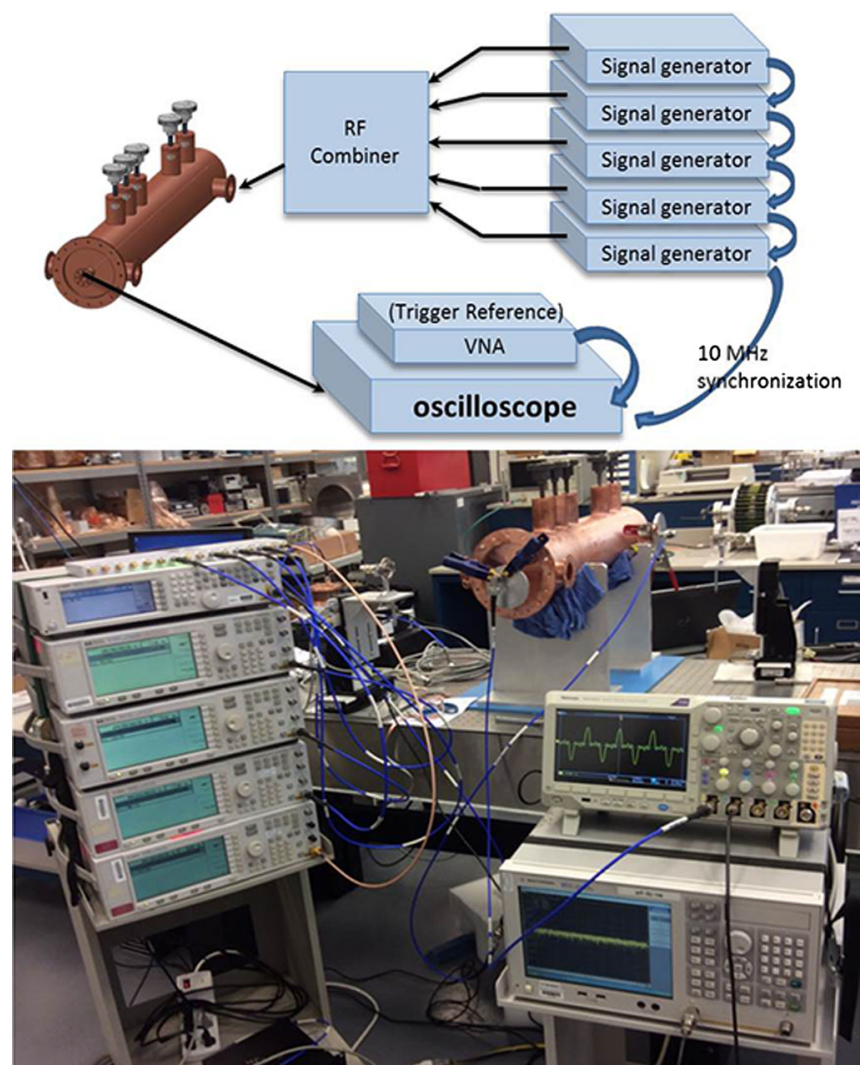

FIG. 21. Schematic drawing (top) and bench set up (bottom) for the mode combination experiment.

combination experiment. Figure 21 shows the schematic and bench setup of this experiment. Five rf signal generators were used to generate the five odd harmonic frequencies (from $95.26 \mathrm{MHz}$ to $857.34 \mathrm{MHz}$ ). The signal generators are synchronized to a $10 \mathrm{MHz}$ reference signal and input to a multichannel rf combiner that feeds the cavity input coupler. An oscilloscope was used to monitor the cavity pickup signal. A synchronized source (Agilent 5071B) was used to generate a 95.26 MHz rf signal as the oscilloscope trigger. The amplitude and phase of each harmonic mode could be adjusted independently and sequentially, in order to fulfill the requirements of the flat-top kicking pulse scheme. The voltage waveform of each harmonic and then the combined signal were directly viewed on the oscilloscope. The mode combination results are shown in Fig. 22 with each mode added in turn starting with the lowest mode. Comparison of the combined kick pulse captured from the oscilloscope display with the simulation is shown in Fig. 23, and the agreement is very good.

Although pickup signal displayed on the oscilloscope is not the exact voltage experienced by the beam, it has demonstrated the principle of combined harmonic modes in the cavity. In practice the relationship between the beam voltage and pick-up signal would need to be calibrated for each mode and the input amplitude corrected accordingly.

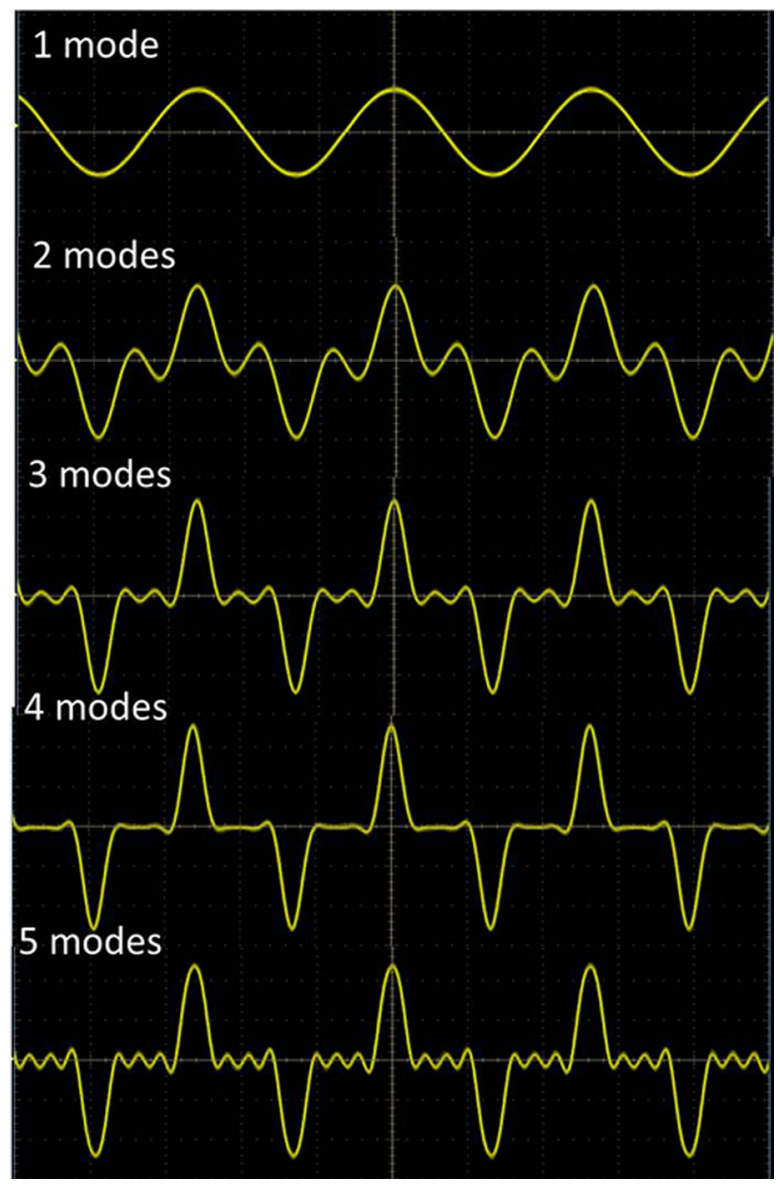

FIG. 22. The result of the odd harmonic modes combined one by one viewed on the oscilloscope display. Here the fifth mode is in the reverse phase to make the final kicking pulse more flat. The final working pulse is a flat-top kicking pulse which is the combination of a DC component and 10 continues harmonic modes separated in four cavities, here we just use five odd harmonic modes in the first cavity for a demonstration purpose.

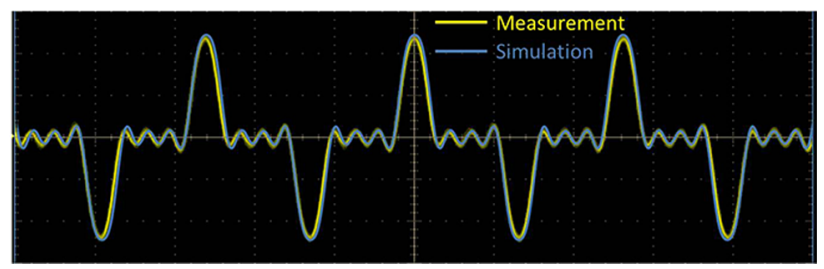

FIG. 23. Comparison of the combined kick pulse from the oscilloscope display image capture with the simulation.

\section{CONCLUSIONS}

An ultrafast rf kicker concept suitable for the JLEIC bunched beam electron cooler ring has been demonstrated. By using four QWR based deflecting cavities, and 10 harmonic modes with the distribution $5: 3: 1: 1$ and an additional DC offset, a flat-top kicking pulse can be synthesized. The cavity was designed and optimized to get high transverse impedances so the sum of the rf wall 
losses in all four cavities is less than $100 \mathrm{~W}$ for a kicking voltage of $55 \mathrm{kV}$. Straight taper segments on the inner conductor of the 5-mode and 3-mode cavities were used to get the higher deflecting modes to be harmonic. Stub tuners were used to compensate the frequency shifts due to the manufacturing tolerances. A single loop input coupler on each cavity can be used on the multimode cavities. By adjusting the loop position and loop size, it is possible to get the fundamental mode of each cavity to be critically coupled and higher harmonics slightly over coupled. Broadband circulators may be considered for the reflected power.

A half scale 5-mode prototype cavity was fabricated in order to validate the electromagnetic characteristics and optimization from the numerical simulations. The frequency shift due to manufacturing tolerances was less than $0.2 \%$ for all modes and could be easily corrected by a combination of the 5 stub tuners. The unloaded quality factors of the five modes were measured and were within $20 \%$ of the calculated values. Bead-pulling measurements using both dielectric and metallic beads moving along the beam axis showed good agreement with the simulated profile of the electric field. Finally an experiment to combine the five modes together, to get the desired kicking pulse was performed. Further optimizations of the cavity structure to reduce the longitudinal E-field component, and investigations into HOM analysis using the wakefield method for high current operation are under way. Real vacuum compatible cavities are planned to be designed and fabricated in the near future to measure the actual kicking effect on an electron beam.

\section{ACKNOWLEDGMENTS}

This work was supported by Jefferson Science Associates, LLC under U.S. DOE Contract No. DEAC05-06OR23177. The authors acknowledge all the assistance received during the construction and bench measurements on this harmonic kicker prototype cavity. We would like to thank Larry Turlington for his help in the design and fabrication process, Steven Castagnola for his help in procuring the materials, Jim Follkie and Teena Harris for their help with the cavity component cleaning, Bill Clemens for the delicate electron beam welding, Damon Combs and Robert Martin on the part machining, Stephen Dutton for his help to made the antenna, and finally Curt Hovater, John Musson and Ramakrishna Bachimanchi for help setting up the mode combination rf experiment.

[1] S. Abeyratne et al., Science requirements and conceptual design for a polarized medium energy electron-ion collider at Jefferson Lab, arXiv:1209.0757.
[2] S. Abeyratne et al., MEIC design summary, arXiv: 1504.07961.

[3] Y. Huang, H. Wang, R. Rimmer, and S. Wang, Beam dynamics analysis for the ultra-fast kicker in circular cooler ring of JLEIC, in Proceedings of the 7th International Particle Accelerator Conference, Busan, Korea (JACoW, Geneva, Switzerland, 2016), p. 510.

[4] Y. Huang, H. Wang, R. A. Rimmer, S. Wang, and J. Guo, Ultrafast harmonic of kicker design and beam dynamics analysis for an energy recovery linac based electron circulator cooler ring, Phys. Rev. Accel. Beams 19, 084201 (2016).

[5] Y. Huang, H. Wang, R. A. Rimmer, and S. Wang, Harmonic resonant kicker design for the MEIC electron circular cooler ring, in Proceedings of the 6th International Particle Accelerator Conference, Richmond, VA, USA (JACoW, Richmond, VA, 2015), p. 2981.

[6] Y. Huang, R. Rimmer, H. Wang, S. Wang, and J. Guo, Ultra-Fast harmonic resonant kicker design for the MEIC electron circular cooler ring, in Proceedings of 56th ICFA Advanced Beam Dynamics Workshop on Energy Recovery Linacs (ERL2015), Stony Brook, NY, USA (JACoW, Stony Brook, NY, 2015).

[7] Y. Huang, H. Wang, R. A. Rimmer, and S. Wang, Taper and tuner scheme of a multi-frequency cavity for the fast kicker resonator in MEIC electron circular cooler ring, in Proceedings of COOL'15 Workshop on Beam Cooling and Related Topics, Newport News, VA, USA (JACoW, Newport News, VA, 2015).

[8] I. Ben-Zvi and J. Brennan, The quarter wave resonator as a superconducting linac element, Nucl. Instrum. Methods Phys. Res. 212, 73 (1983).

[9] B. Xiao, L. Alberty, S. Belomestnykh, I. Ben-Zvi, R. Calaga, C. Cullen, O. Capatina, L. Hammons, Z. Li, C. Marques et al., Design, prototyping, and testing of a compact superconducting double quarter wave crab cavity, Phys. Rev. ST Accel. Beams 18, 041004 (2015).

[10] R. Calaga, I. Ben-Zvi, J. Skaritka, Q. Wu, and B. Xiao, A double quarter-wave deflecting cavity for the LHC, in Proceedings of the 4th International Particle Accelerator Conference, Shanghai, China (JACoW, Shanghai, China, 2013), Vol. 2408.

[11] J. R. Delayen and H. Wang, New compact TEM-type deflecting and crabbing rf structure, Phys. Rev. ST Accel. Beams 12, 062002 (2009).

[12] Microwave Studio, CST-Computer Simulation Technology AG, [www.cst.com].

[13] J. Wlodarczak, P. Glennon, W. Hartung, M. Hodek, M. Johnson, D. Norton, J. Popielarski et al., Power coupler and tuner development for superconducting quarter-wave resonators, in Proceedings of the XXIV Linear Accelerator Conference, Victoria, BC (JACoW, Victoria, 2008).

[14] G. Olry, J.-L. Biarrotte, S. Blivet, S. Bousson, C. Commeaux, C. Joly, T. Junquera, J. Lesrel, E. Roy, H. Saugnac et al., Development of a beta $0.12,88 \mathrm{MHz}$, quarter-wave resonator and its cryomodule for the SPIRAL2 project, Physica C (Amsterdam) 441, 197 (2006).

[15] E. Nissen, A. Hutton, and A. Kimber, A harmonic kicker scheme for the circulator cooler ring in the medium energy electron ion collider, in Proceedings of the 4th 
International Particle Accelerator Conference (IPAC2013), Shanghai, China (JACoW, Shanghai, China, 2013).

[16] D. Proch, J. Sarvas, and E. Somersalo, Computational methods for analyzing electron multipacting in rf structures, Particle Accelerators 59, 107 (1998).

[17] S. Kim, M. Doleans, D. Jeon, and R. Sundelin, Higherorder-mode (HOM) power in elliptical superconducting cavities for intense pulsed proton accelerators, Nucl. Instrum. Methods Phys. Res., Sect. A 492, 1 (2002).

[18] L. C. Maier Jr and J. Slater, Field strength measurements in resonant cavities, J. Appl. Phys. 23, 68 (1952).

[19] J. Lewandowski, G. Bowden, R. Miller, J. Wang et al., Report No. SLAC-PUB-11193, 2005.
[20] H. Hahn, R. Calaga, P. Jain, E. C. Johnson, and W. Xu, HOM identification by bead pulling in the Brookhaven ERL cavity, Nucl. Instrum. Methods Phys. Res., Sect. A 734, 72 (2014).

[21] D. Alesini, A. Falone, M. Migliorati, A. Mostacci, F. Palpini, L. Palumbo, and B. Spataro, Design and RF measurements of an X-band accelerating structure for linearizing the longitudinal emittance at SPARC, Nucl. Instrum. Methods Phys. Res., Sect. A 554, 1 (2005).

[22] P. J. Petersan and S. M. Anlage, Measurement of resonant frequency and quality factor of microwave resonators: Comparison of methods, J. Appl. Phys. 84, 3392 (1998).

[23] National Instruments [www.ni.com/labview]. 